\title{
LA EPISTEMOLOGÍA JURÍDICA Y LAS LLAVES DE LA DEMOCRACIA
}

\author{
LEGAL EPISTEMOLOGY AND THE KEYS TO DEMOCRACY
}

Alessandro Caviglia Marconi ${ }^{1}$

\section{RESUMEN}

El presente trabajo tiene como objetivo mostrar de qué manera el giro hacia la epistemología que se ha operado en la filosofía del derecho, debido a la influencia que ha tenido durante el último siglo y medio el positivismo jurídico, ha producido una desconexión entre dicha disciplina y la filosofía política, trayendo consecuencias importantes tanto en la comprensión como en la práctica del derecho. La mayor de estas consecuencias ha sido que el derecho se ha convertido en una disciplina aislada del resto de disciplinas prácticas, y la práctica jurídica se ha separado de los procedimientos y de la deliberación democráticos. Si la epistemología jurídica asume el giro pragmático sugerido, en su momento, por Oliver Holmes, será posible revertir esta situación y el derecho podrá conectarse nuevamente con las prácticas democráticas.

\section{Palabras clave}

Epistemología jurídica, filosofía política, justicia, positivismo, pragmatismo, democracia

\section{ABSTRACT}

This work aims to show in what way the shift to the epistemology that has taken place in the philosophy of law, due to the influence that legal positivism has had during the last century and a half, has produced a disconnection between said discipline and political philosophy, creating important consequences, both in the comprehension and the practice of law. The major of these consequences is that law has become a discipline isolated from the rest of the practical disciplines, and the legal practice has been separated from the democratic proceedings and the deliberative. If the legal epistemology accepts the pragmatic shift suggested, in its moment, by Oliver Holmes, it will be possible to reverse this situation and law will be able to connect again with democratic practices.

\section{Keywords}

Legal epistemology, political philosophy, justice, positivism, pragmatism, democracy

El siguiente trabajo tiene como objeto reflexionar sobre la epistemología jurídica desde un punto de vista inusual, si tenemos en cuenta la multiplicidad de trabajos que tanto abogados, juristas y filósofos han realizado sobre el tema. El enfoque tradicional de la epistemología jurídica se articula en torno a la pregunta central: ¿cuál es el estatuto epistemológico del derecho en su conjunto o de las normas jurídicas? Para responder a esta preguntase han desarrolladovarias estrategias, entre las que destacan la aproximación epistemológica. Esta aproximación se pregunta por el estatuto de cientificidad del

1 Magister en filosofía por la Pontificia Universidad Católica del Perú. Profesor de Filosofía del Derecho en la Facultad de Derecho de la Universidad de San Martín de Porres, profesor del curso de Interculturalidad y Derechos Humanos en la Maestría en Filosofía de Universidad Antonio Ruiz de Montoya. Además, enseña en la Pontificia Universidad Católica del Perú y en la Escuela de Filosofía de la Universidad Antonio Ruiz de Montoya. Desde el 2001 viene publicando diferentes artículos en libros y en revistas sobre filosofía política, ética, filosofía del derecho, derechos humanos y filosofía de la religión. 
derecho. La segunda perspectiva importante se dirige a los aspectos lógicos del lenguaje normativo propio del derecho. Al respecto, hemos de tener en cuenta dos cosas. La primera es que las investigaciones en ambas perspectivas han dado pie a un sinnúmero de debates fructíferos. La segunda es que al lado de estas dos preocupaciones centrales, se han desarrollado una serie de investigaciones derivadas o complementarias a estas preocupaciones centrales.

El enfoque de este trabajo difiere de estas perspectivas, de tal manera que no faltarán quienes se pregunten si se trata de una contribución a la epistemología jurídica. Y es que el artículo que presento se pregunta por la epistemología del derecho pero desde un punto de vista particular. No se trata aquí de preguntarse el qué de la epistemología jurídica, como quien procura profundizar en la pregunta filosófica por la epistemología jurídica. No se trata de una pregunta metafísica respecto del horizonte de la ciencia del derecho. La pregunta que me interesa formular aquí proviene, más bien, desde la filosofía política y se orienta a investigar cuál es el propósito político y cuáles las consecuencias políticas que tiene el apostar en centrarse en la epistemología jurídica al momento de pensar la filosofía del derecho. Esta pregunta cobra su relevancia debido a que la centralidad en la epistemología jurídica tiene consecuencias políticas importantes, especialmente en relación a la posibilidad de debatir públicamente qué lugar debe tener el derecho, en cuanto actividad profesionalizada y en cuanto disciplina de estudio académico en las universidades, al interior de una sociedad democrática y liberal.

El enfoque del presente trabajo se enmarca en una perspectiva mayor, según la cual la filosofía del derecho (y los temas centrales de dicha disciplina) se deben conectar con la filosofía política, a fin de poder ganar una mayor comprensión. Desde el abandono de la razón práctica durante el siglo XIX, de una parte, y el asenso del neopositivismo, el positivismo lógico y la filosofía analítica durante el siglo $\mathrm{XX}$, por otra, han terminado por producirse dos fenómenos que es necesario destacar. En primer lugar, se operó una desconexión entre la filosofía del derecho y la filosofía política. La autonomía de la filosofía del derecho, respecto de las demás áreas de la filosofía práctica tuvo como una de las causas más relevantes el predominio que adquirió el positivismo jurídico con su afán de cerciorarse de la cientificidad del derecho y los esfuerzos que llevaron a cabo Kelsen y sus seguidores de garantizar que el derecho no se contamine con elementos ideológicos, que provengan de la política, la moral y la religión. En segundo lugar, dicha desconexión terminó por colocar en manos de los juristas y abogados a la filosofía del derecho, en tanto que disciplina académica, de modo que los filósofos tuvieron dos actitudes claramente diferenciadas. Por un lado, un grupo de filósofos abandonaron la reflexión sobre el derecho y se dedicaron a la reflexión sobre la sociedad y a la filosofía política. Esta actitud de un sector de filósofos se vio clara en un sector de la Escuela de Frankfurt, que comenzó a hacer una crítica a las construcciones ideológicas que articulaban las relaciones sociales con el fin de modificar dichas relaciones. El discurso del derecho era considerado como un discurso ideológico más y fue cuestionado, en vez de analizado. De otra parte, otro sector de filósofos se plegaró al trabajo realizado por los juristas y asumió sus principios y metodología de trabajo de manera acrítica. Estos abogados consideraron que hacer filosofía del derecho era dedicarse a lo que los juristas hacían.

Los esfuerzos producidos a partir de las últimas décadas del siglo XX, especialmente, a partir de la publicación de Teoría de la justicia de John Rawls - esfuerzos desarrollados por varios filósofos y juristas destacados, como Ronald Dworkin, Robert Alexy, Jürgen Habermas, entre otros-, no han generado el impacto esperable en relación a la conexión entre la filosofía del derecho y la filosofía política. Esto se debe a que dichas investigaciones fueron interpretadas tanto por juristas y filósofos como trabajos en filosofía política y no en filosofía del derecho. Esta interpretación tiene como base el que los esfuerzos mencionados no se han realizado utilizando la metodología que se supone que es propia de la disciplina. La pregunta que se podría levantar en este punto es la siguiente: ¿es necesaria la identificación 
entre los presupuestos positivistas y la filosofía del derecho? Mi respuesta a dicha pregunta es "no". Pero esta pregunta nos lleva a otra que no carece de interés, a saber, ¿̇es posible utilizar las herramientas de la filosofía práctica para examinar la epistemología jurídica? Mi respuesta es "sî".

\section{El encapsulamiento epistemológico de la filosofía del derecho}

Llama la atención que la filosofía del derecho dirigida por abogados insista en cuestiones epistemológicas, como por ejemplo, en reconocimiento de las normas y los principios jurídicos. Muestra de ello son los diferentes conversatorios, mesas redondas y congresos dedicados a la epistemología del derecho en la que concurren abogados y filósofos. La exacerbación en este punto es la herencia que Hans Kelsen dejó y termina encausando la filosofía del derecho en el lecho del positivismo. Como es sabido, la preocupación de Kelsen es estudiar la teoría del derecho como una ciencia que versa sobre un sistema normativo que se conoce como derecho. La Teoría pura del derecho, si bien el autor austriaco la concibe como una ciencia normativa de la sociedad, en realidad termina presentándose como una ciencia descriptiva de un sistema normativo de la sociedad. De esta manera, Kelsen expresa una desesperación por la delimitación de la ciencia del derecho. En este sentido señala, desde el primer capítulo de la Teoría pura del derecho que:

La Teoría pura del derecho es una teoría del derecho positivo, del derecho positivo en general y no de un derecho particular. Es una teoría del derecho y no una interpretación de tal o cual orden jurídico, nacional o internacional.

Quiere mantenerse como teoría $y$ limitarse a conocer única $y$ exclusivamente, su objeto de estudio. Procura determinar qué es y cómo se forma el derecho, sin preguntarse cómo debería ser o cómo debería formarse. Es una ciencia del derecho y no una política del derecho.
Al calificarse como teoría "pura" indica que entiende construir una ciencia que tenga por único objeto al derecho e ignore todo lo que no corresponda estrictamente a su definición. El principio fundamental de su método es, pues, eliminar de la ciencia del derecho todos los elementos que le son extraños. (Kelsen, 2008, p. 15)

Estas primeras líneas del libro de Kelsen han impreso en el pensamiento jurídico un esfuerzo por garantizar la cientificidad del derecho y han colocado en primer lugar de la discusión en la filosofía del derecho dominante los temas respecto de la epistemología jurídica. Así, temas como de qué manera es posible garantizar la cientificidad del derecho, cómo asegurar que la interpretación jurídica sea científica o de qué manera se puede tener un conocimiento riguroso de la normajurídica (distinguiéndola con exactitud matemática de las normas morales, políticas o religiosas), han acaparado los esfuerzos de los especialistas. Al mismo tiempo, dichos especialistas se ha hecho llamar "iusfilósofos", y llaman a su actividad como "iusfilosofía", a fin de tomar distancia de los filósofos y de la filosofía. Esto responde al afán de destacar que su actividad es diferente de la de los filósofos en dos sentidos específicos. De una parte, es una actividad principalmente de juristas y abogados; mientras que, de otra parte, se trata de una actividad epistemológicamente centrada.

Esta metodología de trabajo ha penetrado, en la actualidad, a dos áreas de relativo reciente interés en el derecho, la argumentación jurídica y el neoconstitucionalismo, desarrollados con cierto interés en España $y$ en Italia respectivamente. En el caso de la argumentación jurídica, se señala que es una forma especial del discurso práctico que adquiere su especificidad en los presupuestos positivistas, a saber, buscar una metodología científica de proceder (el famoso test de proporcionalidad desarrollado por Robert Alexy) y el dirigir la argumentación hacia dentro de los límites de la Constitución y el ordenamiento jurídico y no reflexionar sobre 
los principios constitucionales. En el caso del neoconstitucionalismo, también se retoman las pautas metodológicas del positivismo jurídico al centrar su preocupación en la Constitución y su forma, más que en las consecuencias políticas que se derivan de ella.

Con los términos "iusfilosofía" y "iusfilósofos" se quiere destacar que el enfoque que los abogados le dan a la filosofía del derecho es normativo sobre la disciplina misma, de manera que todo aquél intelectual que no sea abogado y se dedique a la filosofía del derecho, debe de seguir la pauta dada por los abogados. Ya he señalado cómo muchos filósofos han caído en la trampa. Lo que no puedo explicar del todo, aún, es el por qué caen en ella. La hipótesis que puedo esbozar, a esta altura de la investigación es la siguiente: muchos de los filósofos dedicados a la filosofía del derecho son especialistas en epistemología, filosofía de la ciencia o metafísica, pero no en ética ni en filosofía política. De esta manera, carecen de las herramientas conceptuales suficientes para detectar los problemas que tiene el enfoque con el que se comprometen al seguir a los abogados en su empresa.

Otro problema diferente lo ofrece el distinguir (i) qué ha hecho que Kelsen llegue a las conclusiones a las que llegó y (ii) el por qué los juristas siguieron la estela de Kelsen. Respecto de (i) es necesario señalar que la historia del desarrollo del neokantismo y sus ramificaciones, y la versión que dominó en la escuela en la que Kelsen forjó sus ideas dan una pista importante, además de tener en cuenta la posición de dominio que tuvo el positivismo entre fines del siglo XIX y principios del XX. Respecto de (ii) se puede señalar que el impacto que tuvo la vuelta de tuerca que significó el neopositivismo durante el siglo XX y la posición de dominio (en los ámbitos académicos y en la sociedad) en que puso a los abogados el abrazar el positivismo hizo que dicho enfoque se rearticulara y se camuflara a fin de superar las crisis por las que estaba pasando.

De esta manera, la desesperación por tener una descripción correcta del objeto de estudio pervive en todas las formas de reactualización del positivismo jurídico, como es el caso del positivismo metodológico, el neoconstitucionalismo y la llamada Teoría de la argumentación jurídica, que tantos ven como una novedad, cuando en realidad es vino agrio en odres nuevos. Todo esto termina por convertir a la filosofía del derecho en un dar cuenta de mecanismos técnicos que se articulan consistentemente dentro campo del derecho positivo. El cercioramiento de las normas y la coherencia del sistema jurídico, sistema cerrado en sí mismo, se ha convertido en la actividad de los especialistas, que enarbolan la epistemología jurídica como lo nuevo cuando resulta ser un insistir sobre lo mismo.

Esta inflación del cercioramiento hunde sus raíces en la búsqueda cartesiana de abandonar el mar de las dudas para llegar a una Verdad que sea clara y distinta. Se trata de un modo de trabajo intelectual que se vuelca a la certeza absoluta y busca escapar tanto del error como de la duda. Este tipo de razonamiento utiliza un tipo de lógica, la lógica analítica, el cual parte de premisas y a partir de allí llega a conclusiones ciertas, a través de una deducción indudable. El juez Oliver Wendell Holmes Jr. en su conferencia pronunciada el 8 de enero de 1879 en la Facultad de Derecho de la Universidad de Boston cuestionó abiertamente la inflación del cercioramiento en el derecho. Holmes formaba parte del denominado Club de los Metafísicos (Methaphysical Club). El Club de los Metafísicos, fundado en 1872, congregó a intelectuales como Charles Sanders Pierce y William James en Cambridge (Masssachusetts) con el fin de discutir cuestiones filosóficas. También participaron en los intelectuales que llegarían a ser influyentes en la vida norteamericana, como Nicholas St. John Green, Joseph Bangs Warner, John Fiske, Francis Ellingwood Abbot y Chancey Wright. Allí Holmes, quien llegaría a ser un juez famoso de la Corte Suprema de los Estados Unidos entre 1902 y 1932, conoció las ideas pragmatistas que los miembros del club compartían, claro que cada uno a su modo. Holmes conoció y admiró la 
obra de John Dewey, especialmente el libro Experiencia y naturaleza. ${ }^{2}$

En su discurso titulado La senda del derecho Holmes señala con claridad que los juristas se encuentran presos en una falacia que denomina "logicista" que no les permite comprender lo que es el derecho en realidad. De esta manera, señala que:

Podéis asumir -como Hobbes, Bentham o Austin-que todo el Derecho emana del soberano, aunque sea enunciado en primer lugar por los jueces, o podéis pensar que el Derecho es la voz del Zeitgeist, o lo que queráis. Me da lo mismo para mi propósito. Incluso en el caso de que toda decisión requiriese la sanción de un emperador con un poder despótico y una mente caprichosa, aún así nos interesaría, de cara a la formulación de predicciones, descubrir algún orden, alguna explicación racional $y$ algún principio de desarrollo de las normas que aquél estableció. En todo sistema existen tales explicaciones y principios a descubrir. $Y$ en relación con ellos se produce una [....] falacia que juzgo importante exponer.

La falacia a la que me refiero es la idea de que la única fuerza que opera en el desarrollo del Derecho es la lógica. Ciertamente, en el sentido más amplio posible, esa idea resultaría cierta. El presupuesto sobre el que se asienta nuestra forma de pensar el universo es que existe una relación cuantitativa fija entre todo fenómeno y sus antecedentes y consecuentes. Si existiría un fenómeno no sujeto a tales relaciones lo consideraríamos un milagro: algo que se haya al margen de la ley causa-efecto y que trasciende nuestra capacidad de pensamiento o que, al menos, no podemos someter a la razón. La condición que permite nuestra concepción del universo es que éste pueda ser pensado racionalmente $\mathrm{o}$, en otras palabras, que cada uno de sus elementos sea efecto y causa en el mismo sentido en que lo son otros elementos con los que nos hallamos más familiarizados. Del mismo modo, en un sentido muy amplio es cierto que el Derecho responde a un desarrollo lógico, como cualquier otra cosa. El peligro del que hablo no es la admisión de que los principios que gobiernan otros fenómenos también gobiernen el Derecho, sino la idea de que un sistema jurídico determinado -el nuestro, por ejemplo- puede ser elaborado, como las matemáticas, a partir de axiomas generales de nuestra conducta. Éste es el error natural de las escuelas de pensamiento jurídico, pero no se limita a ellas. Una vez oí decir a un eminente juez que nunca emitía una sentencia hasta que estaba absolutamente seguro de que era correcta. Del mismo modo, el disenso judicial es criticado a menudo por entender que simplemente es debido a que uno $u$ otro bando discordantes no habían hecho sus sumas correctamente y que, si se hubieran tomado más trabajo, se habría producido inevitablemente un acuerdo.

Esta forma de pensar es completamente natural. La formación de los abogados es una formación lógica. Los procedimientos lógicos de la analogía, la distinción y la deducción son los más familiares para ellos. El lenguaje de las decisiones judiciales es fundamentalmente el lenguaje de la lógica. Y la forma y el método lógicos satisfacen ese anhelo de certeza y de reposo que se halla en la mente humana. Pero la certeza, generalmente es una ilusión y el reposo no es el destino del hombre. Detrás de la forma lógica subyace un juicio sobre el valor relativo y la importancia de los diversos fundamentos legislativos

2 Respecto del Club de los Metafísicos, cf. BERNSTEIN, Richard; El abuso del mal. La corrupción de la política y la religión desde el 11/9, Bs.As.: Katz, 2006. Pp. 40-43. También, MENAND, Louis; El Club de los Metafísicos. Historia de las ideas en los Estados Unidos, Barcelona: Destino, 2002. Además, WEST, Cornel, The American Evasion of Philosophy. A Genealogy of Pragmatism, The University of Wisconsin Press, 1989, p. 42. 
en competencia. Un juicio a menudo inarticulado e inconsciente, es cierto, pero que constituye la misma raíz y el mismo nervio de todo el procedimiento. Podéis dar una forma lógica a cualquier conclusión. Y siempre podéis suponer la existencia de una condición implícita en un contrato. ¿Por qué podéis presuponerla? Pues a causa de una creencia relativa a la práctica de la comunidad o de un grupo social, a causa de alguna opinión sobre la política jurídica a seguir $o$, en definitiva, a causa de alguna actitud personal sobre determinada materia que no es susceptible de una medición cuantitativa precisa $y$, en consecuencia, que no es susceptible de fundar conclusiones lógicas exactas. Estas cuestiones se constituyen en escenarios de una batalla en la que no existen los instrumentos para realizar determinaciones válidas universalmente y donde la decisión sólo puede expresar la preferencia de un órgano determinado en un tiempo y lugar. No somos plenamente conscientes de hasta qué punto gran parte de nuestro Derecho podría ser objeto de revisión sólo con producirse un ligero cambio en el hábito de la opinión pública. Ninguna proposición concreta es autoevidente, no importa lo proclives que seamos a aceptarla. Ni siquiera la proposición de Herbert Spencer: "todo hombre tiene derecho a hacer lo que quiera a condición de que no interfiera en el mismo derecho de sus semejantes (Holmes, 2012, pp. 68-70)

Esta extensa cita del discurso de Holmes resulta necesaria porque permite comprender con claridad el error del razonamiento que gobierna la mente de los juristas hasta nuestros días y a partir de la cual se elaboran la mayoría de los trabajos en epistemología jurídica. El razonamiento que Holmes denuncia opera partiendo de principios y leyes jurídicos asumidos como axiomas y continúa deduciendo las consecuencias que se derivan de ellos para terminar en el fallo judicial frente a un caso determinado. Cuando se produce una discrepancia en el fallo, este se debe a que alguna de las partes discordantes ha errado al aplicar el procedimiento de deducción. En cambio, de razonar adecuadamente, habrían concordado en el resultado. Esto de debería a que los axiomas de los que se parte son autoevidentes, la mente humana llega a ellos de manera directa, a través de una intuición intelectual, por lo cual no requieren demostración, porque se consideran el punto de partida de toda demostración posible. Se trata de la aplicación del método matemático al derecho, al igual que lo que Descartes procuró hacer con la filosofía. El objetivo de este proceder es llegar a la certeza absoluta, de tal manera, que el proceder de los legisladores y de los jueces tenga exactitud matemática.

Lo que Holmes señala con lucidez es que el supuesto proceder axiomático del derecho no corresponde a la práctica del derecho. Los puntos de partida y los procedimientos que utilizan los juristas y legisladores no son axiomas captados racionalmente, sino se articulan sobre la base de creencias forjadas por la tradición, la historia y el debate en la sociedad. Darle el carácter de axiomas matemáticos tiene como una de sus consecuencias extraer los principios, los procedimientos y los resultados del debate público. En cambio, si comprendemos que los puntos de partidas de la práctica jurídica se encuentra en opiniones forjadas en la sociedad y que pueden ser compartidas por un bando y rechazadas por otro bando, ello nos permite comprender la manera en la que funciona el derecho, especialmente cuando se producen desacuerdos respecto de los fallos judiciales. Tales desacuerdos no se deben a que algunos jueces no han razonado correctamente sino, más bien, a que han partido de creencias fundamentales diferentes. Si esto es así, la tradición imperante en una sociedad puede ser determinante para definir qué es y qué no es derecho. Ahora bien, la tradición puede constituirse de dos maneras: o por el peso de la opinión de las personas e instituciones que tienen poder fáctico, o por la participación libre y abierta de todos los ciudadanos. La deliberación democrática puede ayudar a articular las intuiciones fundamentales de lo que es el derecho en una sociedad. Dicha deliberación, que se procura sea libre de las 
coacciones de los poderes fácticos en la mayor medida posible, permite hacer valer la libertad y la igualdad de los ciudadanos.

Lo que Holmes exige para el derecho es un cambio de mentalidad. Siguiendo a Richard Bernstein, podemos afirmar que este cambio de mentalidad consiste en pasar de una mentalidad volcada a la certeza absoluta a una mentalidad falibilista. Respecto del falibilismo, Bernstein señala que

\section{El falibilismo es la creencia de que cualquier reivindicación de conocimiento, $o$, en general, cualquier reivindicación de validez -incluidas las reivindicaciones morales y politicas-es pasible de análisis, modificación y crítica permanentes (Bernstein, 2006, p. 55)}

De esta manera, el falibilismo pragmático tiene como base una idea respecto de nuestras ideas, la cual sostiene que, en tanto que seres finitos, los seres humanos debemos de revisar nuestras creencias constantemente y no tomarlas de manera dogmática. Esta revisión no significa en caso alguno una defensa débil de nuestras posiciones morales o nuestras creencias sobre la sociedad y el derecho, sino la posibilidad de revisarlas. En cambio, la mentalidad volcada a la certeza absoluta rechaza la finitud humana en el sentido que considera que el ser humano puede llegar a captar la Verdad absoluta, una verdad que no debe someterse a revisión una vez que se ha alcanzado. Esta es la razón por la cual René Descartes señalaba, en sus Meditaciones Metafísicas, que era necesario poner en tela de juicio todas las ideas, per hacerlo sólo una vez en la vida, debido a que una vez que logramos depurar las ideas verdaderas y hemos cumplido con el trabajo filosófico de fundamentar el conocimiento de manera definitiva. Así, una vez que se ha la Verdad absoluta podemos reposar en ella, y simplemente deducir las consecuencias conforme a las reglas del razonamiento correcto. Ello aseguraría el llegar a conclusiones ciertas.
La mentalidad falibilista exige el reconocimiento del pluralismo respecto de las formas de pensar y de concebir la realidad y la sociedad, así como exige el debate público para ajustar las reglas básicas de la convivencia social entre personas que tienen concepciones de la vida religiosas y laicas diferentes. Al asumir la finitud humana y la pluralidad humana, la mentalidad falibilista asume una epistemología particular. Dicha epistemología supone el hecho de que no podemos llegar a una verdad absoluta que se encuentre más allá del mundo de las experiencias humanas y sociales $y$, en vista de la pluralidad humana, debemos de revisar nuestras creencias a través del diálogo y de las nuevas experiencias. De tal manera que la mentalidad falibilista realiza una conexión entre la epistemología, la ética y la política. Es decir, a través de un debate dado en la sociedad, debate que respete exigencias éticas fundamentales, podemos modular el "conocimiento" de la verdad, teniendo en cuenta que dicha verdad puede modificarse en el futuro. Al aplicarse al derecho, la epistemología falibilista exige la conexión entre el derecho y la política.

\section{La desconexión entre el derecho y la política}

Esta epistemoligización de la disciplina jurídica, inspirada en la mentalidad orientada hacia la certeza absoluta, ha operado de manera diferente a la mentalidad falibilista. Así que, en vez de buscar la conexión entre el derecho y la política, ha exigido la desconexión ente ellos. Ya, desde Kelsen, se veía la política como un elemento ideológico que podría contaminar la cientificidad del derecho. Kelsen consideraba a la política como un elemento contaminante para la ciencia del derecho, ciencia que debía de proteger su pureza para mantener su neutralidad y su misma capacidad de erigirse como discurso científico. Aquello que Kelsen, como sus adversarios como Carl Schmitt o Eric Voegelin ${ }^{3}$, entendían por "política" era la política partisana, es decir, aquella actividad que una fracción emprendía para poder ganar

3 Respecto de la posición de Voegelin, Cf. VOEGELIN, Eric; La nueva ciencia política. Una introducción, Bs. As. Katz, 2009.

También véase KELSEN, Hans; ¿Una nueva ciencia política?, Bs. As.: Katz, 2006. 
mayores cuotas de poder. Ni Kelsen ni sus adversarios tenían en mente la concepción liberal de la política, que consiste en la actividad por hacer valer los derechos y las libertades de todos los ciudadanos, en vez de incrementar el margen de dominio de una facción sobre otra.

La desconexión entre en derecho y la política trae consigo una serie de consecuencias importantes. Entre estas quiero destacar tres, una referente a la filosofía del derecho $y$ otras dos que afectan directamente a la democracia. En el campo de la filosofía del derecho, la desconexión condujo a la ya mencionada epistemologización de la materia, con el consecuente efecto de que atrajo hacia la filosofía epistemólogos y a especialistas en metafísica, dejando fuera a los especialistas en ética y en filosofía política. Claro que algunos filósofos, como Habermas o Rawls, especialistas en filosofía política, reflexionaron sobre el derecho, pero su aporte no fue reconocido como filosofía del derecho, sino como filosofía política, debido a que no se centraban de manera exclusiva en cuestiones epistemológicas. Los trabajos de Rawls y Habermas abordan cuestiones epistemológicas respecto al derecho, pero orientan sus reflexiones epistemológicas hacia la filosofía política. El mayor peso relativo que tiene la filosofía política en comparación de la epistemología ha sido la causa por la cual se indique que sus trabajos corresponden a la filosofía política y no a la filosofía del derecho. Es claro que es la mentalidad falibilista la que articula el trabajo de ambos filósofos y que lo que se rechaza desde el grupo que domina el debate en filosofía del derecho es precisamente dicha mentalidad, que termina por subordinar la epistemología a la política y a la ética.

Además, esta distinción trajo consigo dos consecuencias nefastas para la democracia. La primera es la desconexión del derecho con las instituciones y prácticas democráticas. La segunda es la afirmación de Carl Schmitt y sus seguidores, según la cual lo que define el derecho, en última instancia, es la política. Y cuando los schmittianos piensan la política, la ven como política partidaria, tal como ya he afirmado. Quiero concentrarme en la primera reacción. La desconexión del derecho de la dinámica democrática ha traído un fenómeno extraño. Si uno pregunta a los estudiantes de derecho cuál es el fin del derecho, les resulta sorprendente que se les diga que este es asegurar los valores principios políticos de la democracia. Puesto que se encuentran tan acostumbrados a ver en el derecho una herramienta técnica sin remisión a lo político, esta idea les resulta anodina y demasiado "filosófica". Por otra parte, los estudiantes entienden que ese conocimiento técnico los dota de un poder especial, que es el conocimiento de las leyes y procedimientos que rodean la vida de toda persona en la sociedad. El estudio del derecho les brinda todas las llaves de la interacción social. Se trata del poder que se cierne sobre Joseph $\mathrm{K}$. el personaje de la novela El proceso de Kafka, quien, de la noche a la mañana se encuentra inmerso en una pesadilla tejida por la telaraña del derecho. Los abogados tienen todas las llaves porque cuentan con el poder de hilvanar o desarmar la telaraña en la que cualquier persona puede terminar atrapada.

En este punto resulta que la desconexión entre el derecho y la política democrática adquiere su sentido perverso. La filosofía del derecho epistemológicamente centrada se encuentra en una situación singular: o no se da cuenta de las consecuencias políticas que tiene su proceder o intenta retener el poder sobre esas llaves en las manos de los abogados. En el primer caso, se trata de una disciplina que ha perdido la perspectiva debido a su afán de especialización y al excesivo tecnicismo de su lenguaje y pensamiento. En el segundo, se trata de una actividad que conspira contra la misma sociedad democrática a la que el derecho debe de servir como soporte y viabilizador. En esta segunda alternativa, los abogados habrían encontrado la forma para mantener el poder sobre los ciudadanos y desactivar los mecanismos de la sociedad democrática, siendo la epistemologización de la filosofía del derecho un avance en el camino de esa sustracción de poder a la ciudadanía.

Como fuere, esas llaves que el derecho representa, deberían estar en poder de los ciudadanos y no de los operadores jurídicos. Estos han reemplazado a los sacerdotes de la 
Edad Media y a los científicos de los albores de la modernidad. Es por ello que el derecho, desconectado de la dinámica democrática y en manos de los juristas, termina por congraciarse con el poder del neoliberalismo económico. En este sentido, cuando las lleves del derecho se encuentran en manos de los abogados y no en la de los ciudadanos, sucede que el poder que la ley representa genera una sociedad tecnocrática incompatible con los principios de la democracia. La tecnocracia jurídica se cuida de no perder el poder que tiene y es por eso que se vuelve conservadora. En dicho giro conservador, el derecho se conecta con el proyecto neoliberal, a fin de que el rumbo impreso a la dinámica social por parte de los poderes económicos cuenten con el respaldo de un derecho creado a su imagen y semejanza.

Una de las herramientas importantes para hacer que los ciudadanos tengan el poder de esas llaves es realizando la articulación entre la filosofía del derecho y la filosofía política. Se trata de hacer que la filosofía del derecho gire hacia la filosofía política y que el derecho mismo vire hacia la democracia. En este giro político y democrático, las preguntas relevantes de la filosofía del derecho cambian radicalmente. En vez de preguntarse por el estatuto epistemológico de las normas, o sobre la cientificidad del derecho, los filósofos y abogados comienzan a preguntarse por la legitimidad democrática del derecho, por los procesos sociales de deliberación que hacen del derecho lo que es y sobre la manera en la que el derecho puede servir como una herramienta para hacer que tengamos una sociedad más democrática en la que las personas puedan gozar de sus derechos y libertades de manera más plena sin encontrarse sometidos al dominio político, social, cultural y sexual de otras personas dentro de la misma sociedad. Se trata de que los ciudadanos puedan ponerse de acuerdo del lugar y la dirección que debe de tener el derecho por medio de la deliberación pública.

\section{Derecho, sociedad de mercado y democracia}

La desconexión entre el derecho y la política, ha traído consigo una consecuencia perniciosa adicional. Se trata de la penetración del mercado en el derecho. Esta penetración se expresa en la dirección de defensa del mercado que el derecho está tomando. El derecho ha modificado su papel. En vez de defender y promover la democracia, ha terminado por defender y promover el mercado.

No se trata de que el mercado deba suprimir el mercado, sino de garantizar que el mercado tenga su lugar en la sociedad, y que se devore todas las esferas de la sociedad. Desde los años 80, a parir de las políticas económicas establecidas por Ronald Reagan y Margareth Thatcher, estamos asistiendo a una modificación sumamente peligrosa: hemos pasado de una economía de mercado a una sociedad de mercado. Una economía de mercado es aquella que garantiza que exista un espacio de libre intercambio de determinados bienes a través de la libre oferta y demanda. En cambio, una sociedad de mercado es aquella en la cual todos los bienes de la sociedad están expuestos al intercambio en el mercado. ${ }^{4}$

La concentración en la epistemología jurídica propiciada por las reformulaciones del positivismo no solo ha bloqueado la conexión entre el derecho y la política, sino que ha impedido el debate democrático sobre el lugar que debe tener el derecho en la sociedad. Esto ha resultado ser sumamente funcional a las grandes corporaciones mercantiles, debido a que con ello se ha conseguido neutralizar el debate democrático respecto de los límites morales del mercado y se ha bloqueado el cuestionamiento de la sociedad de mercado. De esta manera, el derecho ha ofrecido a los agentes del mercado para bloquear todo debate democrático respecto

4 Cf. SANDEL, Michael; Lo que el dinero no puede comprar. Los límites morales del mercado, Barcelona: Debate, 2013. Además Cf. WALZER, Michael; Las esferas de la justicia. Una defensa del pluralismo y la igualdad, México: FCE, 2001, pp.108-114. 
del sistema dominante. Ello permite que las políticas neoliberales en la economía puedan tener las puertas abiertas y el derecho les dé su bendición.

Pero la colaboración entre el derecho y el neoliberalismo da un giro de tuerca en el llamado Análisis Económico del Derecho. Dicho enfoque ha penetrado profundamente en las escuelas de leyes y representa el acondicionamiento del derecho para que sea una herramienta eficaz para el fortalecimiento de una sociedad de mercado. Bajo la influencia del Análisis Económico del Derecho se ha reemplazando términos como "justicia" o "legalidad" por los de "acuerdo económico satisfactorio" y "eficacia". De esta manera, se propone que las personas puedan canjear derechos -incluso derechos fundamentalespor sumas de dinero. Por ejemplo, una corporación puede comprar el derecho a contaminar el medioambiente o a cazar matar animales en extinción si cuentan con el derecho suficiente para pagar por ellos.

\section{El Análisis Económico del Derecho} concibe los derechos de las personas como mercancías exhibidas en el mercado para el mejor comprador. Así, los derechos de las personas tienen un precio. Y la vida misma también. De hecho hay defensores de este enfoque que sostienen que mantener en vida a una persona puede ser calculado en una cantidad monetaria. Ciertamente, dar calidad de vida a una persona supone una inversión en dinero, pero también supone la presencia de otros bienes no económicos, como el afecto, la amistad, el respeto, entre otros, bienes que no se distribuyen a través del dinero. Sin embargo, en la sociedad de mercado, que el Análisis Económico del Derecho fomenta, tales bienes son puestos en los escaparates del mercado para ser comprados y vendidos. En una sociedad así, hasta el reconocimiento de la dignidad de las personas tiene su precio monetario. Lo único que carece de precio en dicha sociedad es la dignidad misma, pero precisamente por eso es un bien moral desestimado por completo, debido a su insignificancia económica.

\section{Positivismo jurídico, teoría del derecho natural, mercados y autoridad extrapolítica}

La consagración de la sociedad de mercado se ha logrado debido a que a la centralidad de la epistemología en las diferentes disciplinas de pensamiento, tanto en el derecho, en las ciencias sociales y políticas como en la misma filosofía. La centralidad de la epistemología ha logrado fortalecer una autoridad especial, la del especialista. En el derecho se expresa en sus vertientes dominantes. El positivismo jurídico el abogado se convierte en esa autoridad. En la teoría del derecho natural, aquellos que reivindican un acceso al orden natural de las cosas se han convertido en la autoridad. Y, en las ciencias sociales, como en la economía y las ciencias políticas, los especialistas que conocen la técnica y la racionalidad instrumental, se convierten en la autoridad.

Estas autoridades no son autoridades democráticas. Nadie las ha elegido y sus decisiones se encuentran fuera del debate político democrático. Es por ello que se tratan de autoridades extrapolíticas, que en virtud del conocimiento o la técnica que dominan en exclusividad son dotados del poder de definir la suerte de los ciudadanos. En el caso del derecho, los defensores del positivismo sostienen que quien tiene el conocimiento de las leyes y el manejo de su articulación eficaz poseen las llaves de la interacción social. Los defensores de la teoría del derecho natural sostienen que quienes tienen el conocimiento de la naturaleza controlan dichas llaves. Pero, al mismo tiempo, los poderes dominantes en el mercado sostienen lo mismo. Pero, no debemos equivocarnos. No se trata de tres grupos rivales, sino que en el fondo trabajan coordinadamente. El esfuerzo es extraer de la discusión pública los derechos y el lugar que debe tener el mercado en las sociedades contemporáneas. ${ }^{5}$ Como señala con lucidez

Soy deudor de Ronal Reyes respecto de estas ideas. 
Sandel, la consolidación de una sociedad de mercado sucede que los bienes sociales, los derechos y la ciudadanía se corrompen. Los bienes de la sociedad se convierten todos en mercancías, los derecho se convierten en herramientas funcionales al mercado y los ciudadanos se transforman en los engranajes del mercado.

\section{REFERENCIAS}

Bernstein, Richard (2006) El abuso del mal. La corrupción de la política y la religión desde el 11/9, Buenos Aires.: Katz.

Holmes, Oliver Wendell Jr. (2012) La senda del derecho. Madrid: Marcial Pons.

Kelsen, Hans (2008) Teoría pura del derecho. Introducción a la ciencia del derecho. México: Ediciones Coyoacán.

Kelsen, Hans (2006) ¿Una nueva ciencia política? Buenos Aires: Katz.

Menand, Louis (2002) El Club de los Metafísicos. Historia de las ideas en los Estados Unidos. Barcelona: Destino.

Sandel, Michael (2013) Lo que el dinero no puede comprar. Los límites morales del mercado. Barcelona: Debate.

Voegelin, Eric (2009) La nueva ciencia política. Una introducción. Buenos Aires: Katz.

Walzer, Michael (2001) Las esferas de la justicia. Una defensa del pluralismo y la igualdad. México: FCE.

West, Cornel (1989) The American Evasion of Philosophy. A Genealogy of Pragmatism, The University of Wisconsin Press.

Fecha de recepción: 25 de febrero 2015

Fecha de aceptación: 28 de abril 2015 